\title{
Visualization analysis of the study of fund-sponsored clinical nursing papers ${ }^{\dagger}$
}

Xi Tana, Jin-Lian Cheng,

${ }^{a}$ School of Nursing, Shanxi Medical University, Taiyuan, Shanxi 030001 China

${ }^{b}$ First Hospital of Shanxi Medical University, Taiyuan, Shanxi 030001, China

Received: 17 August 2017; Accepted: 11 November 2017; Published: 20 June 2018

Abstract: 0 objective: The aims of this study were to understand the status quo of authors, collaborations between institutions, research hotspots, and research frontiers of fund-sponsored clinical nursing papers and to provide a reference for Chinese scholars to conduct clinica nursing studies in the future.

Methods: The visualization software CiteSpace was used to analyze fund-sponsored clinical nursing papers published between 2012 and 2016 in 12 core journals of nursing.

Results: In the clinical nursing field, there are many cooperative author groups; however, the collaborations between institutions are not close and exist mainly within the same province or city. High-frequency keywords have revealed the four clinical nursing research hotspots of population, diseases, nursing intervention, and others. Chinese medicine nursing, prevention, treatment and nursing, and new technology-aided nursing of ventilator-associated pneumonia are the study frontiers of clinical nursing. Conclusions: Clinical nursing studies are rich in content and cover a wide range of areas, and their hotspots and frontiers are closely related to the requirements of clinical nursing. Collaborations across regions, provinces, and cities are not adequate; there is an urgent need to strengthen the cross-regional exchanges and collaborations to promote the further development of clinical nursing.

Keywords: clinical nursing • fund-sponsored papers • CiteSpace visualization analysis • research hotspot • research frontiers

(c) Shanxi Medical Periodical Press.

\section{Introduction}

The 21st century is an era of "information is value". The rapid development of computer science technology and information engineering technology has created a highly favorable condition for us to effectively extract important information from massive amounts of nursing literature. With the advancement of social progress and the rapid development of technology, the spectrum of disease has undergone great changes. At present, the major cause of human death is associated with daily behaviors and lifestyle, such as chronic diseases, cardiovascular diseases,

${ }^{\dagger}$ This project was supported by scientific research project of the Health Department of Shanxi Province (No. 201301008). and tumors. ${ }^{1}$ The scope of clinical nursing has gradually expanded from the care for patients to a "people-centered" holistic care that takes into account the whole process of human life. In this paper, we used CiteSpace visualization analysis software to intuitively demonstrate the hotspots and frontiers of the fund-sponsored clinical nursing papers in the past 5 years using a mapping knowledge domain method so that we can provide a reference for domestic researchers to conduct clinical nursing studies in the future.

\section{Materials and methods}

\subsection{Data sources}

The fund-sponsored clinical nursing literatures that were published in the core journals of nursing (Chinese 
Journal of Nursing, Chinese Nursing Research, Journal of Nursing Science, Chinese Journal of Practical Nursing, Chinese Journal of Modern Nursing, Journal of Nurses Training, Nursing Journal of the Chinese People's Liberation Army, Journal of Nursing (China), Chinese Nursing Management, Journal of Nursing Administration, Modern Clinical Nursing, and Shanghai Nursing) were used as study subjects. All of the core journals were included in the 2015 edition of "Chinese Science and Technology Journal Citation Reports (Core edition)". We searched a digital journal full-text database (Wanfang database) according to journal titles, and the paper publication time was set between 2012 and 2016. The papers retrieved from searched results were read one by one and were manually screened and evaluated strictly according to inclusion and exclusion criteria. Ultimately, 7742 fund-sponsored clinical nursing papers were included as the sample data for this study.

\subsection{Literature inclusion and exclusion criteria}

Fund-sponsored clinical nursing papers related to basic nursing, health assessment, internal medicine care, surgical care, obstetric and gynecological care, pediatric care, otorhinolaryngological care, dermatological care, emergency care, intensive care, psychological care, Chinese medicine care, humanistic care, other clinical nursing theory and technology, and novel medical technology-aided care were included. Papers discussing nursing education; nursing management; care provided outside of the hospital, such as stricken area, community, and home care; knowledge links; tips; seminars; minutes; notices; humanistic excellence; book reviews; and other column literature were excluded.

\subsection{Methods}

The visualization software CiteSpace, which was developed by Dr. Chaomei Chen at Drexel University in the United States, was used in this study. The 7742 included literatures were selected through the Wanfang database, and the bibliographical reference data were exported in the RefWorks plain text format. The data were imported into the CiteSpace software, the literature publication time selected was between 2012 and 2016, and the time interval was set as 1 year. The selected node types were author, institution, and keywords. The top 50 data entries that had the highest frequencies of node type of various time intervals were selected, the default value was selected for term sources, the minimum spanning tree simplification algorithm was selected for map pruning, the visualization view was the classical view, and the mapping knowledge domains were shown after running. The calculated data were exported through the backstage of CiteSpace, and the results were analyzed together with the map.

\section{Results}

\subsection{Analysis of authors' academic collaborations}

There was a total of 183 nodes $(\mathrm{N})$ and 112 links (E) in the mapping knowledge domains of author collaborations (Figure 1), with a density of 0.0067 . In the figure, each node represents an author, the size of the node represents the publication frequency of the author, and the thickness of the circle is positively proportional to the publication frequency of the corresponding year. The number of lines represents the collaborations between authors, and the thicknesses of the lines represent the closeness of the collaborations between authors. Yan Hu, Qixia Jiang, and Weili Wang occupy relatively important node weights in the author co-occurrence map. Through the connection lines between nodes, we can intuitively observe that there are apparent collaborations between authors in the clinical nursing field. Yan $\mathrm{Hu}$, Zhenqi Lu, Huiling Li, Yuxia Zhang, and Ying Gu form the first author collaboration group, with Yan $\mathrm{Hu}$ as the center. Qixia Jiang, Qing Peng, Xin Zhou, and Li Liu form the second author collaboration group, with Qixia Jiang as the center. Weili Wang and Jingfang Hong form the third author collaboration group, with Weili Wang as the center. In addition, the map indicates that small academic groups with fewer collaborators are scattered around.

\subsection{Analysis of collaborations between research institutions}

There were a total of 120 nodes and 66 links in the mapping knowledge domains of the collaborations between institutions (Figure 2), with a density of 0.0092 . In the map, each node represents an institution, and the larger the node, the more the papers published by the institution. The number of lines represents the collaboration between the institutions, and the thicknesses of the lines represent the closeness of the collaborations between the institutions. The institution whose frequency was ranked at first was the School of Nursing of Fudan University, followed by the First Hospital of Shanxi Medical University and the School of Nursing of Anhui Medical University. A total of 28 research institutions had more than 20 publications, and the institutions were distributed in 13 provinces, cities, and autonomous regions. In terms of region, Jiangsu province had the most, with eight institutions, followed by Shanghai, with five 


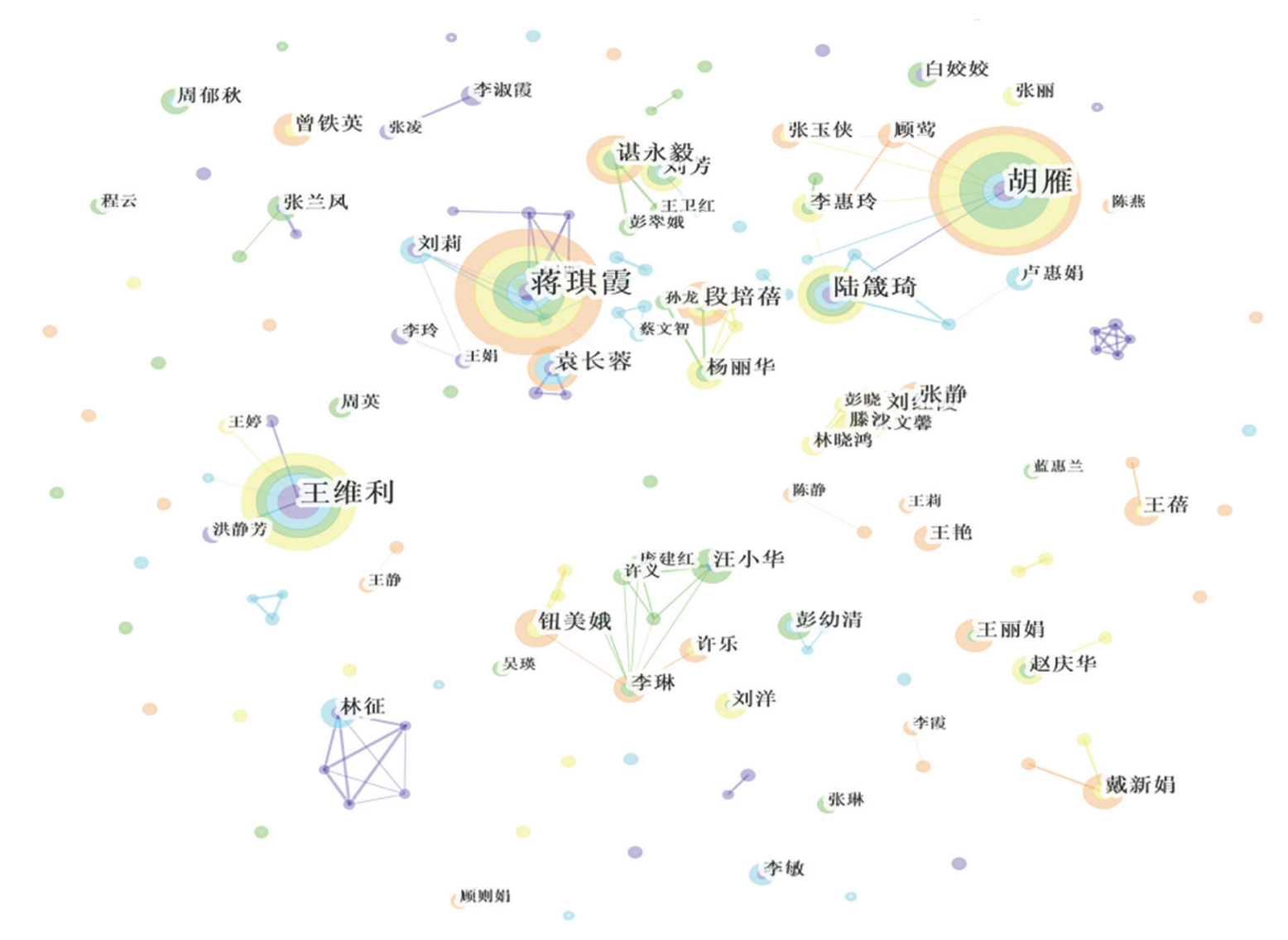

Figure 1. The mapping knowledge domains of author collaborations.

institutions; Guangdong province, with three institutions; and Hubei province and Guangxi Zhuang autonomous region, each with two institutions; Shanxi province, Anhui province, Heilongjiang province, Sichuan province, and Henan province and the municipalities of Chongqing, Beijing, and Tianjin had one institution each. In terms of the types of institutions, among the 28 listed, 11 were medical schools, accounting for $39.29 \%$, and 17 were hospitals, accounting for $60.71 \%$; 16 of the 17 hospitals were affiliated to medical schools (Table 1).

In terms of collaborations between institutions, there were apparent collaborations between the School of Nursing of Fudan University, the School of Nursing of Nanjing University, the Nursing College of Nantong University, the Children's Hospital of Fudan University, Fudan University Shanghai Cancer Center, Huadong Hospital of Fudan University, the First Affiliated Hospital of Nanjing Medical University, and Nanjing Drum Tower Hospital, the Affiliated Hospital of Nanjing University Medical School. The Second Military Medical University had apparent collaborations with the School of Nursing of Shanghai University of Traditional Chinese Medicine, the Sixth Affiliated People's Hospital of Shanghai Jiao Tong University, and Harbin Medical University Daqing Campus. There were collaborations between the School of Nursing of Nanjing University of Chinese Medicine, Jiangsu Province Hospital of Traditional Chinese Medicine, and Nanjing General Hospital of the Nanjing Military Region. In addition, some institutions that scattered around with more publications were mainly medical colleges, which had collaborations with their affiliated hospitals, such as Shanxi Medical University and the First Hospital of Shanxi Medical University, the School of Nursing of Sun Yat-Sen University, and the First Affiliated Hospital of Sun Yat-Sen University.

\subsection{Research hotspots}

Keyword analysis is a word frequency analysis method that can be used to determine the hotspots, knowledge structure, and development trends of certain research fields. ${ }^{2}$ There were a total of 100 nodes and 211 links in the co-occurrence mapping knowledge domains of keywords (Figure 3 ), with a density of 0.0426 . In the map, larger nodes suggest the higher co-occurrence frequency of a given keyword and also indicate that 


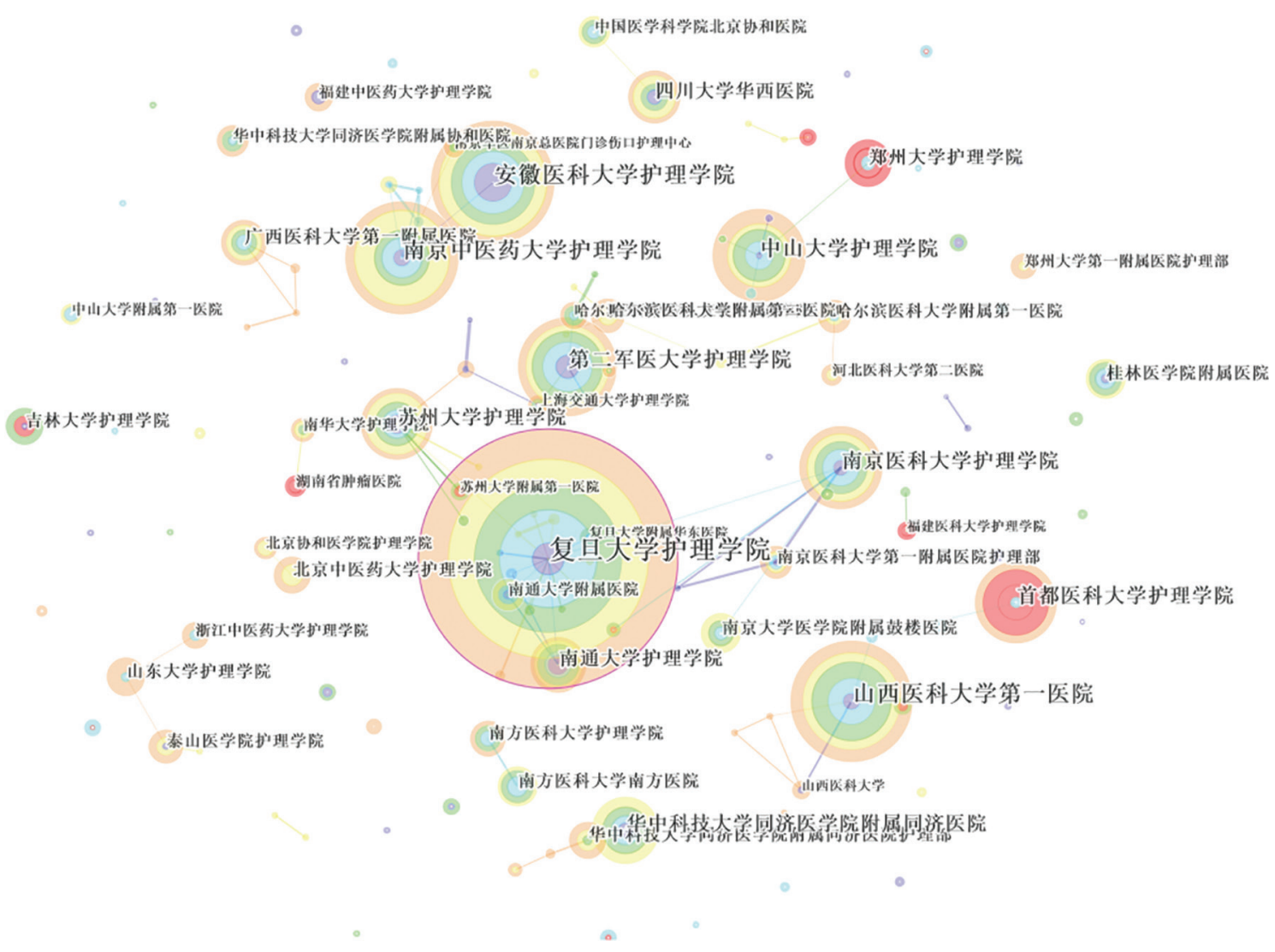

Figure 2. Mapping knowledge domains of collaborations among institutions.

\begin{tabular}{clc}
\hline Rank & Name of institution & Frequency \\
\hline \hline 1 & School of Nursing of Fudan University & 126 \\
2 & First Hospital of Shanxi Medical University & 61 \\
3 & School of Nursing of Anhui Medical University & 61 \\
4 & Tongji Hospital of Tongji Medical College of Huazhong University of Science and Technology & 61 \\
5 & School of Nursing of Nanjing University of Chinese Medicine & 57 \\
6 & School of Nursing of the Second Military Medical University & 50 \\
7 & The First Affiliated Hospital with Nanjing Medical University & 48 \\
8 & School of Nursing of Sun Yat-Sen University & 47 \\
9 & School of Nursing of Nanjing Medical University & 42 \\
10 & Capital Medical University School of Nursing & 41 \\
11 & School of Nursing of Soochow University & 36 \\
12 & Union Hospital of Tongji Medical College of Huazhong University of Science and Technology & 35 \\
13 & Nanjing General Hospital of Nanjing Military Region & 34 \\
14 & The Second Affiliated Hospital of Harbin Medical University & 33 \\
15 & Nursing College of Nantong University & 29 \\
16 & West China Hospital of Sichuan University & 27 \\
17 & Fudan University Shanghai Cancer Center & 26 \\
\hline
\end{tabular}

Table 1. Statistics of publication frequencies of research institutions. 


\begin{tabular}{lll}
\hline Rank & Name of institution & \\
\hline \hline 18 & The Nursing College of Zhengzhou University & 24 \\
19 & The First Affiliated Hospital of Soochow University & 23 \\
20 & The First Affiliated Hospital of Guangxi Medical University & 23 \\
21 & The First Affiliated Hospital of Sun Yat-Sen University & 23 \\
22 & Huadong Hospital of Fudan University & 22 \\
23 & The First Affiliated Hospital of Chongqing Medical University & 21 \\
24 & Affiliated Hospital of Guilin Medical University & 21 \\
25 & Tianjin University of Traditional Chinese Medicine & 21 \\
27 & Nanfang Hospital of Southern Medical University & 21 \\
28 & The Sixth Affiliated People's Hospital of Shanghai Jiao Tong University & 20 \\
\hline
\end{tabular}

Table 1. (Continued).

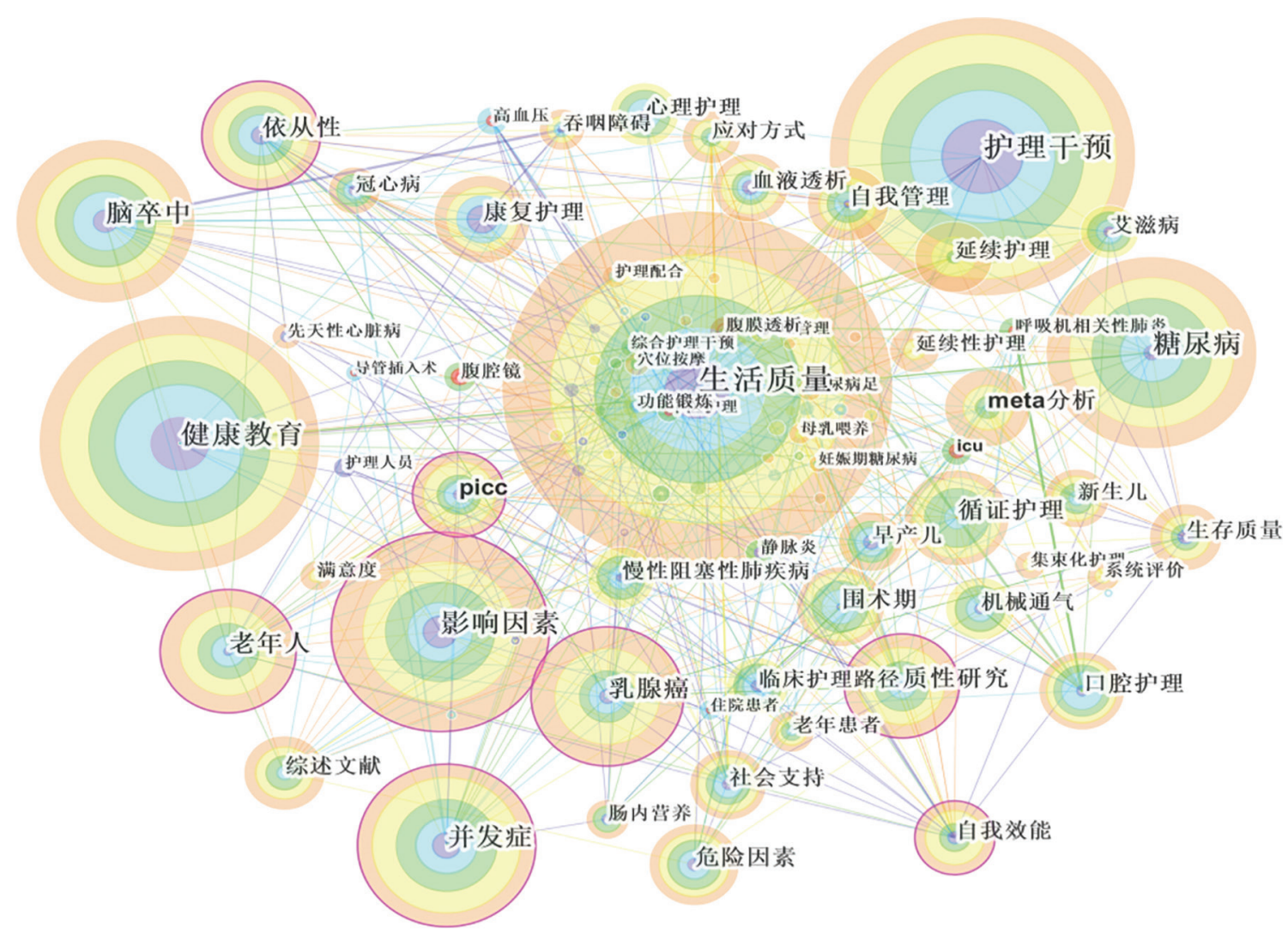

Figure 3. Co-occurrence mapping knowledge domains of keywords.

the study objective of the literature associated with the keyword is a hotspot of clinical nursing research. From the map, it can be found that a total of 46 keywords appear 20 or more times. "Quality of life" has the highest frequency, with 273 occurrences. Keywords with occurrence frequencies of more than 100 times include "quality of life", "nursing intervention", "health education", "influencing factor", "diabetes", "the elderly", "stroke", "complications", and "breast cancer". In the mapping knowledge domains, the meanings of certain keywords are extremely close or they are different expressions of synonyms; these words should be merged, including "the elderly" and "the elderly patients" and "extended care" and "continuity of care". After merging similar words and exporting data backstage, the results are shown in Table 2. 


\begin{tabular}{|c|c|}
\hline Keyword & Frequency \\
\hline Quality of life & 273 \\
\hline Nursing intervention & 217 \\
\hline Health education & 196 \\
\hline Influencing factor & 154 \\
\hline Diabetes & 150 \\
\hline The elderly & 129 \\
\hline Stroke & 127 \\
\hline Complications & 125 \\
\hline Breast cancer & 107 \\
\hline Extended care & 91 \\
\hline Compliance & 85 \\
\hline Qualitative research & 82 \\
\hline Evidence-based care & 75 \\
\hline Rehabilitation care & 70 \\
\hline PICC & 68 \\
\hline Risk factor & 64 \\
\hline Perioperative & 64 \\
\hline Oral care & 63 \\
\hline Self-management & 61 \\
\hline Meta-analysis & 61 \\
\hline Review literature & 59 \\
\hline Self-efficacy & 58 \\
\hline Social support & 56 \\
\hline Hemodialysis & 56 \\
\hline Quality of life & 54 \\
\hline Psychological care & 50 \\
\hline Chronic obstructive pulmonary disease & 49 \\
\hline Mechanical ventilation & 49 \\
\hline Premature baby & 48 \\
\hline New born & 46 \\
\hline Clinical nursing path & 45 \\
\hline Coping style & 42 \\
\hline AIDS & 41 \\
\hline Coronary heart disease & 39 \\
\hline Dysphagia & 34 \\
\hline Enteral nutrition & 32 \\
\hline Laparoscopy & 25 \\
\hline Systematic assessment & 23 \\
\hline Degree of satisfaction & 23 \\
\hline Hypertension & 22 \\
\hline Clustered nursing & 22 \\
\hline Ventilator-associated pneumonia & 22 \\
\hline Peritoneal dialysis & 21 \\
\hline ICU & 21 \\
\hline Phlebitis & 21 \\
\hline Functional exercise & 20 \\
\hline
\end{tabular}

Table 2. Statistics of high-frequency keywords in clinical nursing.

\begin{tabular}{lcc}
\hline Keyword & Burst degree & Year \\
\hline \hline Catheterization & 4.57 & 2012 \\
Laparoscopy & 3.15 & 2012 \\
Ventilator-associated pneumonia & 6.53 & 2013 \\
Chinese medicine care & 4.74 & 2013 \\
Peritoneal dialysis & 5.61 & 2014 \\
\hline
\end{tabular}

Table 3. Burst degree and burst year of keywords.

\subsection{Analysis of research frontiers}

The CiteSpace software was used to investigate the keywords, and five bursting keywords were identified: "laparoscopy", "catheterization", "ventilator-associated pneumonia", "Chinese medicine care", and "peritoneal dialysis" (Table 3).

\section{Discussion \\ 4.1. Analysis of author distribution and collaboration}

The type of study that uses visualization means to describe author structure has become the forefront of bibliometrics and has begun to attract the attention of scientific researchers. The in-depth analysis of high-frequency authors in the clinical nursing field and the analysis of the associations between authors with close collaborations allow the investigation of the research themes and academic thoughts of the collaboration groups. The collaboration groups of Yan Hu mainly come from the School of Nursing of Fudan University, Fudan University Shanghai Cancer Center, and the Children's Hospital of Fudan University. By searching relevant works and the literature, a certain academic association between the authors in the collaboration group was noted. Professor Yan $\mathrm{Hu}$ and Zhenqi Lu undertook the chief editorial work of the monograph "Practical Cancer Care"; Yuxia Zhang and Ying Gu participated in the writing of "Pediatric Nursing (bilingual)", which was edited by Professor Yan Hu; and Ying Gu participated in the writing of "The Theory and Practice of Evidence-based Nursing", which was edited by Yan Hu. The research projects of Professor Yan $\mathrm{Hu}$ included the study of evidence-based nursing, evidence translation, and a series of studies on improving the quality of life of breast cancer patients. In recent years, Yan $\mathrm{Hu}$ has been the editor of "Evidencedbased Nursing", "Nursing Research", "Practical Cancer Care", and other teaching materials and has had a great influence in the field of clinical nursing research. By searching for other authors in the collaboration 
group, the themes of the literature published by the collaboration group were identified as mainly cancer care, evidenced-based nursing practice, and pediatric care, including studies of social support and quality of life for breast cancer and acquired immune deficiency syndrome (AIDS) patients, the care for children with congenital heart disease, and premature infant- and other pediatric-related care.

Qixia Jiang edited and published the monographs "Pressure Ulcer Nursing", "A Practical Guide for the Prediction and Prevention of Pressure Ulcers in Adults", and "A Clinical Practical Guide of Wound Care". Her nursing research projects are mainly related to wound care. Some time ago, she began studying various methods for pressure ulcer-based wound care, and she has rich experience in treating various wounds; in particular, she conducted an in-depth study on the diagnosis and treatment of pressure ulcers, diabetic foot ulcers, traumatic ulcers, and postoperative wounds that are difficult to heal and introduced the negative pressure wound therapy concept. The institutions of the authors of the Qixia Jiang collaboration group mainly include Nanjing General Hospital of Nanjing Military Command and Jiangsu Provincial Hospital of Traditional Chinese Medicine, and the themes of papers published through collaborations mainly include prevention and treatment of pressure ulcers, prevention and treatment of diabetic foot ulcers, and the diagnosis and treatment of other chronic wounds that are difficult to heal. The published papers described wound treatment, infection prevention, wet therapy, vacuum-assisted closure, and other treatment methods.

Professor Weili Wang serves as the dean of the School of Nursing of Anhui Medical University and serves as the chief editor or associate editor of "Theory and Practice of Psychological Nursing", "Clinical Nutrition", "Introduction to Nursing", "Thinking and Communication", "Interpersonal Relationship and Communication", and other teaching materials. The institutions of the authors in his collaboration group mainly include the School of Nursing of Anhui Medical University. The papers published by the collaboration group of Professor Weili Wang mainly cover digestive system cancer care and gynecologic and breast cancer care, which include the quality of life, self-efficacy, psychological distress, coping styles, therapeutic communication, and other related content of cancer patients. In addition, there are scattered collaborations between authors in the map; there are fewer authors in Professor Wang's collaboration group, and the collaborations are mainly within the same institution or between the Medical University and its affiliated hospitals.

\subsection{Analysis of the distribution of institutions and their collaborative relationships}

The quantity and quality of research papers are important scales to measure the scientific research level, performance, and strength of researchers. ${ }^{3}$ Among the top 28 institutions ranked by their publication numbers, the institutions with frequencies more than 20 can be divided into two major categories: hospitals and medical universities, to a certain extent because the subjects of this study are clinical care papers. There are 120 institution nodes in the map, while there are only 66 collaboration connection lines between them, suggesting that the collaborations between institutions were not very close and that the collaborations were scattered, rather than forming a large collaboration network. The collaborations were mainly regional within a certain area, and cross-province and cross-city collaborations between institutions were rare.

\subsection{Analysis of research hotspots}

The research hotspot in a field refers to the topics and views that are discussed in a large number of research papers with the same theme in a certain period of time. ${ }^{4}$ Keywords are an important component of the literature: relevant literature in certain directions can be searched through keywords, and the study focus of the literature can also be understood through the keywords. Therefore, the network knowledge domains formed by the CiteSpace software analyzed the co-occurrence frequencies of the same groups of keywords in the literature. From the sizes of the nodes in the map, we can intuitively identify the scales of the occurrence frequencies of keywords in clinical nursing studies in the past 5 years, which represent how much attention is paid to the contents of these keywords in a certain period of time. Thus, the hotspots and foci of nursing research in a certain period of time can be obtained through keyword analysis. By merging similar words, the following four hotspots in clinical nursing research were ultimately classified and summarized.

\subsubsection{Research hotspot of population}

In the past 5 years, the hotspots of research population were the elderly, newborns, and premature infants. As the aging of China's population continues to increase, the number of elderly patients and the types of diseases keep increasing, and the challenges and requirements brought to clinical nursing become increasingly significant. The "Outline of the 13th Five-Year Plan for the National Economic and Social Development of the 
People's Republic of China"5 emphasizes that aging science research should be strengthened to meet the physical and mental needs of the elderly; therefore, elderly care has attracted more and more attention. With the increase in age, the health conditions of the elderly change, characterized as declines in immunity, high prevalence of medical conditions, increases in chronic disease patients, and gradual loss of daily living abilities. ${ }^{6}$ In China, the hotspot of elderly nursing research mainly focuses on the assessment and prevention of elderly patient falls, pressure ulcers, accidental inhalation and other risks, mental health care, improvement of the quality of life of elderly patients, and strengthening the self-management of elderly patients. Yu et al. ${ }^{7}$ effectively reduced the incidence of elderly patient falls through comprehensive nursing intervention. Guo et al. ${ }^{8}$ described the factors affecting the quality of life of elderly patients and reviewed the intervention measures applied to patients from the aspects of cognition, behavior, and psychology. Facing this aging situation, at this stage, we should vigorously promote care for the elderly and continue improving elderly care-related norms and service guidelines. We should keep the elderly in mind, providing comprehensive, systematic, holistic care for them so that they may age healthily.

In the field of nursing, with the implementation of the two-child policy, the need for pediatric care services is increasing. The hotspots of these studies focus on disease screening of newborns and nutrition support of premature babies. Premature infants are an extremely vulnerable population, and it is highly important for the medical staff to provide premature babies with good medical care to improve their survival rate and their future quality of life..$^{9}$ Chinese scholars ${ }^{10-12}$ have been investigating and studying the optimal feeding method suitable for premature infants to promote their growth and development. The development of pediatric medical care services is related to the development of the nation, economy, and society. Nursing research focusing on pediatrics has gradually become a research hotspot, promoting the development of pediatric health care.

\subsubsection{Research hotspot of diseases}

In the past 5 years, cardiovascular and cerebrovascular diseases, diabetes, breast cancer, AIDS, and respiratory system diseases have been included in the hotspot diseases of clinical nursing research. With the rapid development of the society, people's life and working styles have undergone tremendous changes, which, together with the changes in psychological states and environmental factors, have caused disease profiles to change: the prevalence and mortality rates of cardiovascular and cerebrovascular diseases, malignant tumors, diabetes, respiratory system diseases, and other chronic, noninfectious diseases have gradually increased. The "Outline of the 13th Five-Year Plan for the National Economic and Social Development of the People's Republic of China" also emphasizes effective prevention and control of such chronic, non-infectious diseases and the control of the AIDS epidemic at a low prevalence level. ${ }^{5}$

The epidemic trend of cardiovascular disease risk factors is obvious, with its increasing incidence over the years. ${ }^{13}$ In 2014, the mortality rate of cardiovascular disease in China topped those of several other diseases and was even higher than those for tumors and other chronic non-infectious diseases. ${ }^{14}$ The risk factors of cardiovascular disease include diabetes, hypertension, and dyslipidemia. The prevention and treatment of cardiovascular disease, the nursing interventions for risk factors, and the psychological care of patients are the current hotspot issues studied by nursing staff.

Diabetes prevalence has shown an increasing trend among younger patients and has become one of the diseases greatly endangering human health. According to the statistical data of the World Health Organization, by 2030 , the number of diabetic patients in the world will reach 439 million. ${ }^{15}$ Facing such a serious situation, strategies to effectively prevent and treat diabetes constitute the research focus of the nursing field. In the past 5 years, diabetes care has mainly focused on health education for diabetes patients and the improvement of patient self-management. The content of health education for diabetes patients included the integrated modes of health education, structured education, implementation of self-management education projects, complication simulation experience education, and peer scene health education.

Breast cancer is a type of malignant tumor that endangers women's physical and mental health. Its incidence and mortality rates are both ranked first among women's malignant tumors. ${ }^{16}$ The treatment process for breast cancer is long, as patients must undergo surgery, radiotherapy, chemotherapy, and other comprehensive treatments during the disease course. Furthermore, there is a possibility of recurrence, which can result in negative physiological and psychological impacts on patients that seriously affect their quality of life. Psychological care plays a key role for breast cancer patients in improving their confidence and body function. Implementing targeted psychological interventions and perioperative care for breast cancer patients, helping them with postoperative functional exercises, and helping them to rebuild their confidence are the key projects currently attracting attention in the nursing field. 
AIDS, caused by human immunodeficiency virus infection, is a chronic infectious disease with an extremely high mortality rate that has become a public health issue threatening human health. ${ }^{17}$ Since 1985 , when the first AIDS patient was identified in China, the medical care field has fostered studies on the prevention and treatment of AIDS and the care for afflicted patients. With the development of prevention and treatment protocols, AIDS care is maturing. At present, AIDS care mainly focuses on the care for AIDS complications, psychological care, social support, and patient treatment compliance. Improving the quality of life of AIDS patients has become an important goal for treatment and care. ${ }^{18}$

The threat of respiratory system diseases to human health has gradually attracted attention. In the past 5 years, the respiratory system diseases that have attracted more attention are chronic obstructive pulmonary disease and ventilator-associated pneumonia. Ventilator-associated pneumonia is the most common complication of patients on mechanical ventilation; thus, preventing and treating ventilator-associated pneumonia and improving its prognosis are key components of clinical nursing. The research hotspot of clinical nursing mainly focuses on respiratory tract management and oral care. Improving the pulmonary function of patients with chronic obstructive pulmonary disease and improving the quality of life of patients are also research hotspots of current clinical nursing.

\subsubsection{Research hotspot of nursing intervention}

The research hotspot of disease nursing focuses on evidence-based nursing, psychological nursing, health education, rehabilitation nursing, and continuous nursing. With the formation of the holistic nursing concept, the content of nursing service has gradually extended to long-term care, chronic disease management, and other aspects, to comprehensively provide professional care, psychological support, and rehabilitation nursing for patients, along with various other tasks to meet patients' physiological, psychological, and social health needs from various aspects and at various levels.

The essence of evidence-based nursing is to use the best evidence as the basis for making clinical nursing decisions. With the development of nursing, it has become an accepted practice to provide care for patients only based on experience and intuition. Evidence-based nursing has attracted more and more attention, as it allows the clinical nursing staff to give full play to their subjective initiative, can promote the development of nursing theory and techniques, and can ensure the scientific nature of the nursing service; at present, it has been widely applied clinically. As many diseases are closely related to psychological factors, patients with chronic diseases or critical illnesses are prone to experiencing anxiety, fear, and other psychological responses. Thus, psychological nursing has gradually attracted the attention of scholars and has become an important component of clinical nursing. Currently, it mainly focuses on providing psychological care for tumor patients. Health education can help patients to establish healthy lifestyles and behaviors. In clinical nursing work, providing targeted rehabilitation care and health education can increase the patient's awareness of treatment and their compliance with treatment so that they can recover normal physiological function as soon as possible.

\subsubsection{Other research hotspots}

More attention has been paid to patients' quality of life, compliance, self-management, self-efficacy, and social support. With the increase in chronic diseases, the study of quality of life has attracted the attention of nursing professionals, and its practical significance in clinical treatment and nursing has been gradually revealed. Interventions by self-management measures can change patient behaviors so that the patient's health condition can be maintained in a satisfactory state, and self-management can mobilize the patient's subjective initiative. The self-care management model has gradually begun to receive attention. In addition, performing targeted social support projects can effectively improve a patient's treatment compliance, quality of life, self-efficacy, and self-management ability. ${ }^{18}$

\subsection{Analysis of the themes of clinical research frontiers in China}

Burst word refers to a term for which the occurrence frequency increases faster over a shorter period of time or the growth rate of its usage frequency increases significantly. Burst words have the property of dynamic change and can accurately reflect the frontiers of the relevant discipline. ${ }^{19}$ In this study, we used the burst word detection technology of CiteSpace software, the knowledge domains, and bursting keywords to discover research frontiers in the clinical nursing field.

By analyzing visualization maps and burst words, the development trends of research in the clinical nursing field can be obtained. In 2012, the burst words were "laparoscopy" and "catheterization". Laparoscopic surgery, as a relatively new surgical procedure, is different from traditional surgery. It has the advantages of less 
trauma, less pain, quick recovery, and low recurrence rate and has been widely used in the diagnosis and treatment of diseases. ${ }^{20}$ Intravascular catheterization is now widely used clinically and has provided an ideal channel for patients for infusion, parenteral nutrition, tumor chemotherapy, thrombolysis, and others. With the clinical application of new medical technologies, laparoscopy, and catheterization-aided care, complication prevention care and perioperative care have gradually become the new research trends. In 2013, the burst words were "ventilator-associated pneumonia" and "Chinese medicine care". The "National Nursing Profession Development Plan (Year 2016-2020)"21 emphasizes that Chinese medicine should actively provide dialectical care and specialized care with traditional Chinese medicine characteristics, innovate the Chinese medicine care model, improve the Chinese medicine care level 21 , and make a full play of Chinese medicine care in disease treatment and rehabilitation promotion. In addition, with the wide use of ventilators, preventing and treating ventilator-associated pneumonia have become keys of clinical nursing. These findings suggest that studies on Chinese medicine care and ventilator-associated pneumonia care have been further developed. In 2014, the burst word was "peritoneal dialysis", due to the aging trend of the population and the increase in the number of patients with uremia caused by various diseases over the years; the burst word represents an important

\section{References}

1. Chai YY, Zhang XY, Li Y. Influential factors of cause of death for principal diseases in China. Chin Gen Pract. 2010;13:1025-1027 (in Chinese).

2. Chang Q, Duan ZG, Zheng JZ, et al. Literature review of Chinese Medical Malpractice with CiteSpace II. Med Philos (A). 2015;36:78-81 (in Chinese).

3. $\mathrm{Li} \mathrm{YL,} \mathrm{Wu} \mathrm{XJ.} \mathrm{Bibliometric} \mathrm{analysis} \mathrm{of} \mathrm{the} \mathrm{literature}$ on grading systems of nursing care published from 2009 through 2013 in China. J Nurs Sci. 2014;2:710 (in Chinese).

4. Liu YY. Analysis of the Hotspots, Frontier Topics and Academic Groups of the Study on College Entrance Examination of China Since the 21st Century. Liaoning Normal University; 2014 (in Chinese).

5. Xinhua net. Outline of the 13th five-year plan for the national economic and social development of the People's Republic of China; 2016. http://www.gov.cn/xinwen/2016-03/17/content_5054992.htm. Accessed November 18, 2016 (in Chinese). treatment method for the advanced stages of renal disease, and diet management, complication prevention, treatment, and care of peritoneal dialysis patients will become the development trends of the clinical nursing field in the future.

\section{Conclusions}

Clinical nursing involves a large amount of publications covering a wide range of subjects. Collaborations between authors are more common than between institutions, but the collaborations are mainly within the same institution or between the medical university and its affiliated hospitals; collaborations across provinces, cities, and regions are rare. In the future, there will be an urgent need to strengthen cross-region exchanges to achieve mutually beneficial cooperation. With the changes in disease profiles and people's life and working styles, in the past 5 years, the research hotspots in clinical nursing have mainly focused on key issues attracting national and social attention. Chinese medicine care; the prevention, treatment, and care of ventilator-associated pneumonia; and new technology-aided care have become the emerging research topics in clinical nursing.

\section{Conflicts of interest}

All contributing authors declare no conflicts of interest.

6. Sun SY, Zeng H, Zhang LP. Population aging and present nursing conditions and countermeasures in China. J Nurs Sci. 2006;11:76-78 (in Chinese).

7. Yu LJ, Fang Z, Yang CJ, et al. Influence of comprehensive nursing interventions on knowledge, attitude and practice of fall prevention among elderly patients. J Nurs Sci. 2013;6:13-15 (in Chinese).

8. Guo ZT, Liu XD, Zhao YH. Research progress on the influence of nursing intervention on the quality of life of old people. Chin Nurs Res. 2012;26:18251826 (in Chinese).

9. Yin LJ. New advances in nursing care of premature infants. China Pract Med. 2010;35:234-235 (in Chinese).

10. Zhang XN, Zhou CM, Zhou Y, et al. The influences of different feeding methods on the feeding tolerance and growth of preterm infants. $J$ Nurse Train. 2012;20:1829-1831 (in Chinese).

11. Ren XD, Sun XM, Peng Y, Shen QQ. Clinical observation on intestinal tract comfort care in preventing 
feeding intolerance for premature infants. Chin Nurs Res. 2013;1:56-58 (in Chinese).

12. Chi CX, Hou HP, Zeng JF, Li WD. Enteral feeding methods for premature and low birth weight infants. Modern Clin Nurs. 2012;10:10-12 (in Chinese).

13. Chen WW, Gao RL, Liu LS, et al. Summary of the 2013 cardiovascular disease report of China. Chin Circ J. 2014;7:487-491 (in Chinese).

14. Sui $H$, Chen WW, Wang W. Interpretation of the main points of the 2015 cardiovascular disease report of China. Chin J Cardiovasc Med. 2016;21:259-261 (in Chinese).

15. Narayan KMV, Williams D, Gregg EW, Cowie CC. Diabetes Public Health: From Data to Policy. London: Oxford University Press; 2011.

16. Huang HY, Li XM, Zhang XR, Yang CY. Progress in perioperative nursing of breast cancer. Hainan Med J. 2011;22:137-138 (in Chinese).

17. Zhao EJ, Cui D, Liang SY, Guo JL, Wang YM, Lu WQ. Study on the epidemic situation and pre- ventive measures of AIDS. Modern Prev Med. 2012;39:1597-1599 (in Chinese).

18. Cai MX, Sheng Y. Research progress in online social support for people living with HIVIAIDS. J Nurs Adm. 2016;12:851-853 (in Chinese).

19. Shi JY. Mapping Knowledge Domains Analysis of Transfusion Medicine Research Based on CiteSpace III. The Fourth Military Medical University; 2015 (in Chinese).

20. Zhang SX. Nursing coordination of ectopic pregnancy laparoscopic surgery. China Med Herald. 2011;9:100-101 (in Chinese).

21. National Health and Family Planning Commission of the People's Republic of China. The notice of the publication of the National Nursing Profession Development Plan (Year 2016-2020); 2016. http://www.moh.gov.cn/yzygj/ s3593/201611/92b2e8f8cc644a899e9d0fd572 aefef3.shtml. Accessed November 18, 2016 (in Chinese).

How to cite this article: Tan X, Cheng JL, Li YJ. Visualization analysis of the study of fund-sponsored clinical nursing papers. Front Nurs. 2018; 2: 127-138. https://doi.org/10.2478/fon-2018-0017 